\title{
Liquid crystal alignment induced by micron-scale patterned surfaces
}

\author{
E. Willman, L. Seddon, M. Osman, A. Bulak, R. James, S. E. Day, and F. A. Fernandez, \\ University College London, London WC1E 6BT, United Kingdom
}

(Received 20 January 2014; published 1 May 2014)

\begin{abstract}
Induced bulk orientation of nematic liquid crystal in contact with micron-scale patterned surfaces is investigated using the Landau-de Gennes theory by means of three-dimensional simulations. The effect of the size and spacing of square cross-sectional well and post patterns is investigated and shown to influence the orientation of the liquid crystal bulk, far removed from the surface. Additionally, the effective anchoring strength of the induced alignment is estimated using a modified version of the torque balance method. Both azimuthal and zenithal multistability are shown to exist within unique ranges of feature sizes.
\end{abstract}

DOI: 10.1103/PhysRevE.89.052501

PACS number(s): 61.30.Hn, 61.30.Pq

\section{INTRODUCTION}

Most liquid crystal devices depend on the aligning influence of surfaces for their operation. Liquid crystal (LC) molecules interact with a surface in a complex way governed by a number of physical and chemical processes, including Van der Waals and polar forces and steric effects [1-3].

Traditionally, controlled anchoring has been achieved using a number of different processes, for example, rubbing, photoalignment, oblique evaporation of $\mathrm{SiO}$, or using surfactants [4]. An alternative approach that has received some interest involves patterning the aligning surfaces with either topographic relief features or flat micron-scale regions imposing different LC orientations, e.g., checkerboard or stripe patterns alternating between horizontal and vertical alignment. Techniques used for patterning the surfaces include AFM inscription [5], photolithography [6], nanoimprinting [7], and micro and nanoprinting of chemical alignment patterns [8-11].

Irrespective of the underlying mechanisms responsible for anchoring, its effect on a macroscopic scale can be described by a phenomenological surface energy term added to the free energy at interfaces between liquid crystal materials and aligning surfaces. A commonly used surface energy term was proposed by Rapini and Papoular [12] and takes the form $\frac{W}{2} \sin ^{2} \alpha$, where $W$ is the strength of the anchoring and $\alpha$ is the angle the director makes with the direction favored by the surface or easy direction. Modifications and extensions to this formulation have been introduced in the past, allowing, for example, the description of anisotropic anchoring surfaces [13-15]. Nevertheless, the assumption of a simple $\sin ^{2} \alpha$ type of variation in the energy remains popular.

In the case of alignment by topographically patterned surfaces, the patterns typically consist of elongated ridges (1D) or discrete protruding or depressed features (2D) [7,16-18]. Locally, the patterned surfaces are either treated with surfactant to achieve homeotropic anchoring $[17,19,20]$ or left untreated, whereby planar degenerate anchoring is assumed $[18,21,22]$. Distortions arise in the liquid crystal director field in the vicinity of the surface features. However, in the absence of other aligning effects, for example, electric fields or other alignment surfaces, a uniform and well-defined orientation is observed in the liquid crystal bulk at some

*a.fernandez@ucl.ac.uk distance above the patterned surface. This can be considered an effective anchoring plane with characteristics (such as an effective anchoring energy) that depend on the shape, size, and arrangement of the underlying patterned surface.

It is known that some patterned surfaces that induce large distortions are capable of supporting multiple stable anchoring directions. Bistable display devices relying on this property have been developed [16,17]. Switching between the anchoring states in these devices is achieved using monopolar electric fields that interact with the flexoelectric polarization present in many LC materials $[23,24]$. The concept of an effective surface energy has been used in Ref. [25] to predict the phase behavor of bistable LC cells containing one patterned surface.

Theoretical studies on the anchoring effect induced by patterned surfaces have been made in the past. Depending on the shape and dimensions of the surface patterns, different liquid crystal configurations occur and different approaches are adequate: In the case of smooth and shallow surface patterns, giving rise to continuous, defect-free, director fields the Oseen-Frank elastic theory [26,27] is sufficient. In the special case of shallow sinusoidal gratings, the anchoring properties have been studied using analytic methods $[28,29]$, whereas numerical methods are required for more complex shapes [30,31]. More pronounced patterned surfaces induce large distortions in the director field, leading to defects. In this case, one must resort to the Landau-de Gennes $Q$-tensor theory in conjunction with numerical methods if defects are to be treated accurately [32-34]. In cases with sufficiently small patterns, molecular simulations have also been used to examine the anchoring effect and LC orientation near the patterns $[11,35]$.

In the references above, director configurations in the vicinity of wells or posts have been modelled separately in the past, and the duality of the two cases was suggested by Kitson et al. [6]; however, to our knowledge, a unified study of them and their effect on the bulk alignment has not been presented to date. In this paper comprehensive three-dimensional modeling is used to study the liquid crystal structure induced by surfaces patterned with posts or wells of rounded square cross section and of various sizes and separations.

\section{NUMERICAL MODEL}

Equilibrium configurations of the liquid crystal are found by minimizing the Landau-de Gennes free-energy functional 
using the finite-element method over a mesh of tetrahedra $[32,33]$. The liquid crystal is described by an order tensor ( $Q$-tensor) representation that takes into account the head-tail symmetry of the nematic director and allows for order parameter variations and biaxiality. The $Q$ tensor is a symmetric and traceless rank 2 tensor with five independent components that can be written as

$$
Q_{i j}=\frac{S}{2}\left(3 n_{i} n_{j}-\delta_{i j}\right)+P\left(k_{i} k_{j}-l_{i} l_{j}\right),
$$

where the indices $i, j$ run from 1 to $3, \delta_{i j}$ is the Kroenecker delta, $S$ is the scalar order parameter, and $P$ is a biaxiality parameter which is normally zero in the bulk of the liquid crystal. The three orthogonal vectors $n, k$, and $l$ are the eigenvectors of $Q$. The eigenvector $n$, corresponding to the largest eigenvalue $S$, is the nematic director.

In the Landau-de Gennes formalism the total free energy in the liquid crystal domain $\Omega$, in contact with a surface $\Gamma$, is

$$
F_{\text {total }}=\int_{\Omega}\left(f_{B}+f_{D}\right) \mathrm{d} \Omega+\int_{\Gamma} f_{S} d \Gamma,
$$

where $f_{B}$ is the thermotropic bulk energy density, $f_{D}$ is the elastic distortion energy density, and $f_{S}$ is the surface energy density. The thermotropic energy density given by

$$
f_{B}=\frac{A}{2} \operatorname{tr}\left(Q^{2}\right)+\frac{B}{3} \operatorname{tr}\left(Q^{3}\right)+\frac{C}{4} \operatorname{tr}\left(Q^{4}\right)
$$

is minimized when the liquid crystal order parameter $S=S_{0}$, the equilibrium order parameter. The elastic energy contribution $f_{D}$ is

$$
f_{D}=\frac{1}{2}\left\{L_{1} Q_{i j, k} Q_{i j, k}+L_{2} Q_{i j, k} Q_{i k, k}+L_{6} Q_{l k} Q_{i j, l} Q_{i j, k}\right\},
$$

where the commas in the subscript represent derivatives with respect to the spatial coordinate that follows, and summation over repeated indices is assumed. The coefficients $L_{1}, L_{2}$, and $L_{6}$ are material parameters that can be related to the Frank [26,27] splay, twist, and bend constants $K_{11}, K_{22}$, and $K_{33}$ [36,37]. The anchoring at the interface between the solid surface and the liquid crystal material is modelled by the following expression for the surface energy density:

$$
f_{S}=a \operatorname{tr}\left(Q^{2}\right)+W_{1}\left(v_{1} \cdot Q \cdot v_{1}\right)+W_{2}\left(v_{2} \cdot Q \cdot v_{2}\right),
$$

where $v_{1}$ and $v_{2}$ are unit vectors and $W_{1}$ and $W_{2}$ are anchoring strength coefficients. These two unit vectors and the preferred liquid crystal director orientation at the surface are mutually orthogonal. The $\operatorname{tr}\left(Q^{2}\right)$ term is an additional isotropic term that stabilizes the surface order parameter. Setting $a=\left(W_{1}+W_{2}\right) /\left(6 S_{s}\right)$ favors a surface order parameter equal to a value $S_{s}$, whereas omitting it causes the linear terms to drive the surface order towards $\pm \infty$ [15]. In the present case, planar degenerate anchoring is assumed over the patterned surface. This is achieved by setting $W_{2}=0$ and $W_{1}>0$, with $v_{1}$ parallel to the local surface normal. This results in an energy term with approximately a $\frac{W_{1} 3 S_{0}}{4} \sin ^{2} \alpha$ variation with respect to the difference between the local tilt angle and the easy direction, and quadratic variation with respect to the surface order tensor. (a)

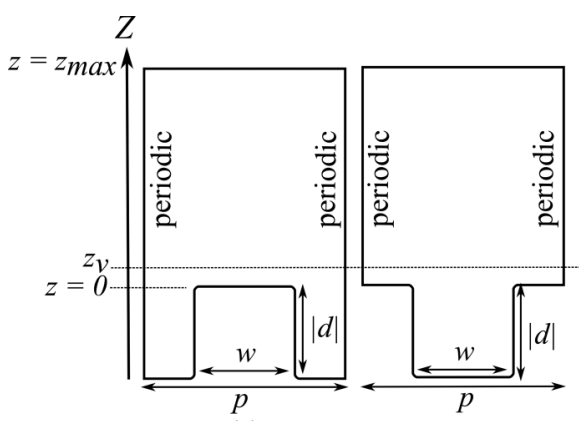

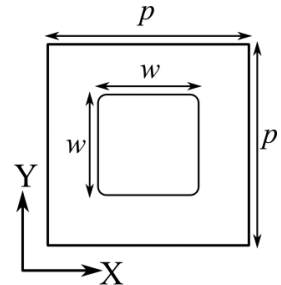

(c)
FIG. 1. Schematic diagram showing the geometries of unit cells representing surfaces covered in periodic post and well structures. $x-z$ or $y-z$ planes for (a) posts, (b) wells, and (c) the $x-y$ plane for both posts and wells.

\section{A. Details of the calculation cell}

Two types of regularly spaced surface features are considered in these calculations: posts and wells of different height (depth) and spacing. Figure 1 shows schematic diagrams of the cross sections of the $3 \mathrm{D}$ calculation window.

The shape of the posts or wells is defined by the following function:

$$
\begin{aligned}
f(x, y)= & d 1-\left[\frac{1}{1+e^{s(x+w / 2)}}-\frac{1}{1+e^{s(x-w / 2)}}\right] \\
& \left.\times\left[\frac{1}{1+e^{s(y+w / 2)}}-\frac{1}{1+e^{s(y-w / 2)}}\right]\right\},
\end{aligned}
$$

where $d$ is depth of a well $(d<0)$ or the height of a post $(d>$ $0)$. The width and period pitch of the features are determined by $w$ and $p$, respectively, and $s$ determines the sharpness of edges and corners of the features. In all cases presented here, $s=230$ was used, giving rise to rounded corners with a radius of approximately $20 \mathrm{~nm}$.

Meshes of tetrahedral elements were used in the finiteelement calculations using first-order Lagrange elements, with a higher node density near the bottom surface where large distortions in the director field occur and lower near the top where the director field is more uniform. Mesh sizes ranging from a few thousand nodes to over 100000 nodes were used, depending on the feature height and size of the structure considered, to achieve mesh independence. Periodic boundary conditions were imposed on all lateral surfaces to simulate an extended patterned surface.

Referring to Fig. 1, the highest point of the bottom surface, that is, the top of a post or the rim level of a well, is chosen as the reference value $z=0$. The top surface of the modeling window is located high enough to be well above the region where the LC orientation becomes uniform when no anchoring on this surface is applied. Typically, this corresponds to a few microns for a pitch, $p$, of $800 \mathrm{~nm}$.

The anchoring of the liquid crystal on the bottom surface, including the surface of the posts and wells is assumed planar degenerate, with a zenithal anchoring strength sufficiently strong to prevent topological transitions through anchoring breaking. Using the representation of the anchoring energy (5) described in Ref. [15], a value of $W_{1}=1.5 \times 10^{-3} \mathrm{~J} / \mathrm{m}$ was 
TABLE I. Values of elastic coefficients used in the modeling.

\begin{tabular}{lccc}
\hline \hline & $K_{11}(\mathrm{pN})$ & $K_{22}(\mathrm{pN})$ & $K_{33}(\mathrm{pN})$ \\
\hline $5 \mathrm{CB}$ & 6.2 & 3.9 & 8.2 \\
Modified & 7.0 & 3.9 & 7.0 \\
\hline \hline
\end{tabular}

used to describe the zenithal anchoring strength, while a value of $W_{2}=0$ characterizes the planar degeneracy. The value of $a$ is set to $a=W_{1} / 6 S_{0}$ to keep the surface order parameter close to $S_{0}$. The liquid crystal alignment on the top surface either is left free or is fixed at different strengths, as described in the later sections.

The values for the thermotropic coefficients used are those of 4-cyano-4'-pentylbiphenyl (5CB) at $1.4^{\circ}$ degrees below the clearing temperature: $A=-0.12 \times 10^{6} \mathrm{Nm}^{2}, B=-2.13 \times$ $10^{6} \mathrm{Nm}^{2}$, and $C=1.73 \times 10^{6} \mathrm{Nm}^{2}$ [38]. In the calculations, two different sets of elastic coefficients are used. For the first set (corresponding to the 5CB liquid crystal material [39]) the relationship $K_{22}<K_{11}<=K_{33}$ holds, while for the second set $K_{22}<K_{11}=K_{33}$ is assumed. The values used are given in Table I.

\section{RESULTS}

The macroscopic anchoring and bulk alignment properties of surfaces patterned with small wells or posts were investigated using the model described above. In particular, the effect of the size and separation of the surface feature on the induced bulk orientation was investigated.

Starting the simulations from two different initial bulk LC configurations, one with low tilt and the other with high tilt, for both posts and wells structures we find two stable, topologically unique configurations that occur for different ranges of values of $d / w$ : one with no defects, or continuous, and the other containing a pair of $\pm 1 / 2$ defects in the director filed or discontinuous. The continuous configuration can also have two different forms, a symmetric one that exists only when the height or the depth of the surface structures are very small compared to their width and an asymmetric one. The discontinuous or defect structure is always asymmetric. Figure 2 shows schematically some of these cases. A region of bistability is also observed for a range of values of the aspect ratio $d / w$, where the initial low-tilt and high-tilt bulk LC configurations relax into stable continuous (both symmetric and asymmetric) and defect states, respectively. In this case, the transition between the continuous and defect state passes through intermediate states of higher energy and it is not spontaneous. Outside this region of $d / w$ values, only one of these states is reached irrespective of the initial starting configuration.

As can be seen in Fig. 2, the continuous symmetric configuration [Fig. 2(c)] leads to a horizontal alignment in the bulk of the liquid crystal above the surface features. Both asymmetric states, with and without defects, induce an orientation in the bulk tilted to a degree that depends on the aspect ratio of the surface features and on the period of the arrangement. On planes parallel to the bottom surface and closely above the top of the surface features, the tilt varies

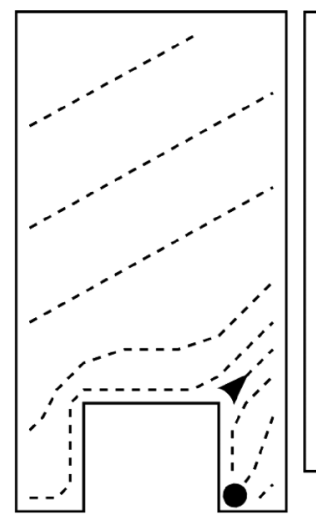

(a)

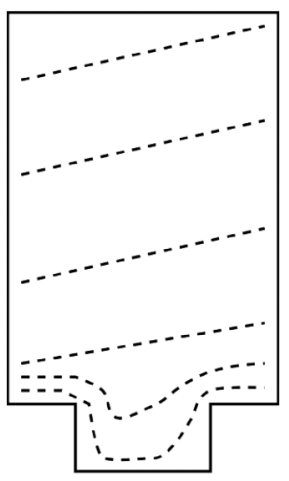

(d)

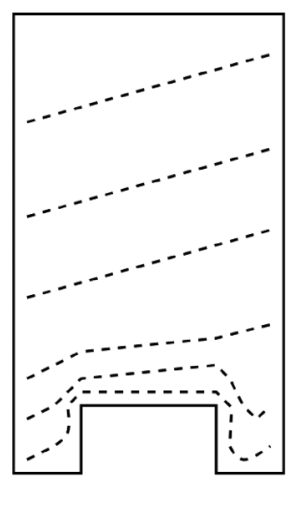

(b)

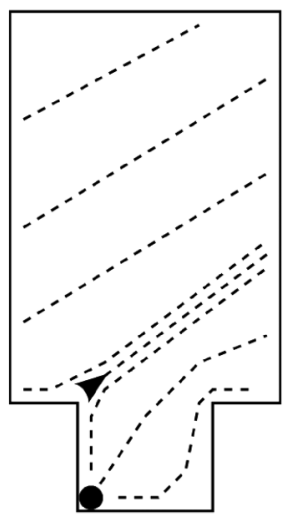

(e)

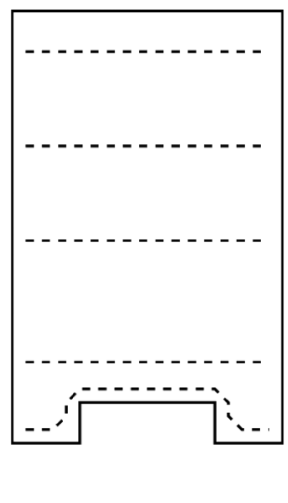

(c)
FIG. 2. Stable director configurations for posts and wells. [(a) and (e)] Discontinuous (asymmetric), [(b) and (d)] continuous asymmetric, and [(c)] continuous symmetric. (f) The $x-y$ cross section. The round markers indicate position of defects of $+1 / 2$ strength, whereas triangles indicate $-1 / 2$ defects.

over the posts or wells but this variation rapidly disappears as the distance from the top of the features is increased. The azimuthal alignment is oriented along the direction of one of the diagonals of the cross section of the surface features [Fig. 2(f)] and because of the symmetry of the structures, the asymmetric states will have a twofold degeneracy. Similarly, the induced tilt will have two possible directions for each azimuthal orientation. Figure 3 shows the tilt variations and average tilt angle as a function of the distance from the top of the surface features for the case of posts of aspect ratio of $d / w=1.875$ and a width to separation ratio $p / w=2$ ( $d=750 \mathrm{~nm}, w=400 \mathrm{~nm}, p=800 \mathrm{~nm}$ ) when using the material parameters of $5 \mathrm{CB}$ and no anchoring is imposed on the top surface of a thick cell. It can be seen that the transition from a rapidly varying LC orientation to a uniform one in the bulk occurs within a thin layer near the surface features. Consequently, a virtual anchoring surface can be defined at a small distance above the surface features, where the induced liquid crystal tilt is sufficiently uniform, as indicated by $z=z_{v}$ in Fig. 1. Above this surface the tilt remains constant; this is the easy direction imposed by the patterned surface on the bulk liquid crystal orientation.

Figure 4 shows the tilt angle in the bulk of the liquid crystal (assuming no anchoring on the top surface) as a function of 


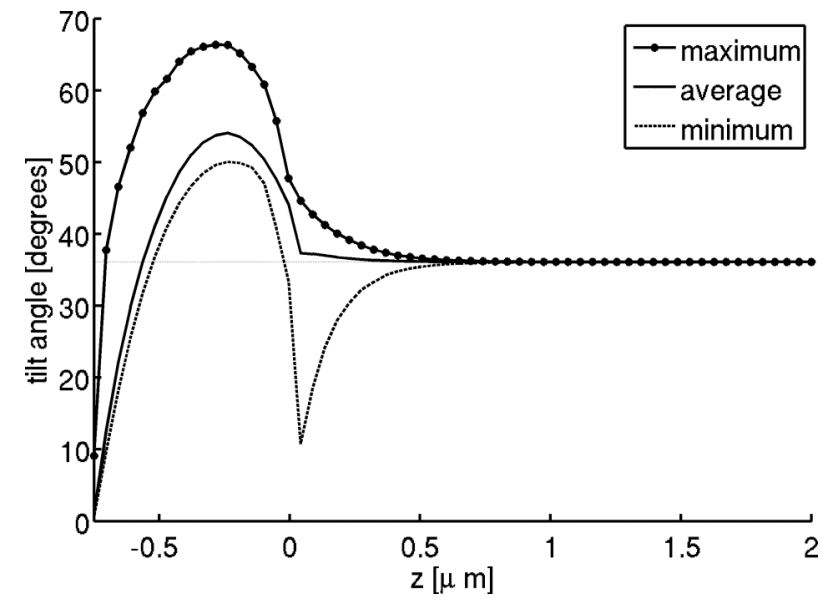

FIG. 3. Tilt in the bulk above the top of the surface features as a function of $z$. This example is for 5CB and an array of posts with $d=750 \mathrm{~nm}, w=400 \mathrm{~nm}$, and $p=800 \mathrm{~nm}$.

the ratio between the height (depth) to the width of posts and wells. The calculations are for periodic arrays of posts (or wells) with a ratio between spacing and width $p / w=2$ and using the material parameters of $5 \mathrm{CB}$. The feature width and period are fixed at $w=400$ and $p=800 \mathrm{~nm}$, respectively, while the feature height is varied between positive and negative values (posts and wells). Arrays of short posts result in a symmetric continuous director field configuration inducing zero bulk tilt angle. As the post height is increased, a region of bistability is reached where a stable tilted configuration with defect pairs appears when the simulation is started from a high-tilt initial LC configuration and the continuous symmetric state when starting from a low-tilt initial LC configuration. As the post height is further increased, the continuous symmetric configuration becomes asymmetric, resulting in a finite bulk tilt angle. For large values of the post height, only the defect state is stable irrespective of the starting bulk LC configuration.

In the case of wells the situation slightly differs. While the continuous symmetric configuration is stable for very

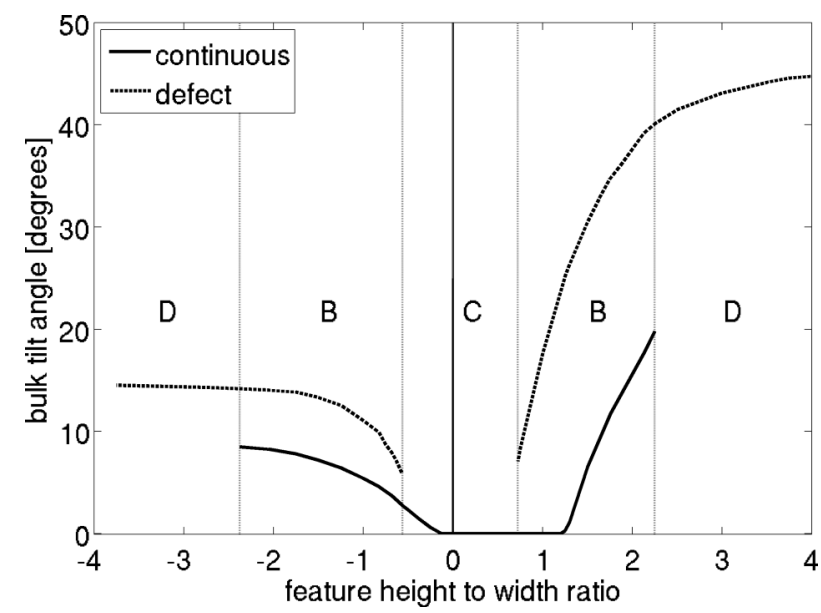

FIG. 4. Effective bulk tilt angle as a function of the feature aspect ratio $(d / w)$ for $5 \mathrm{CB}$.

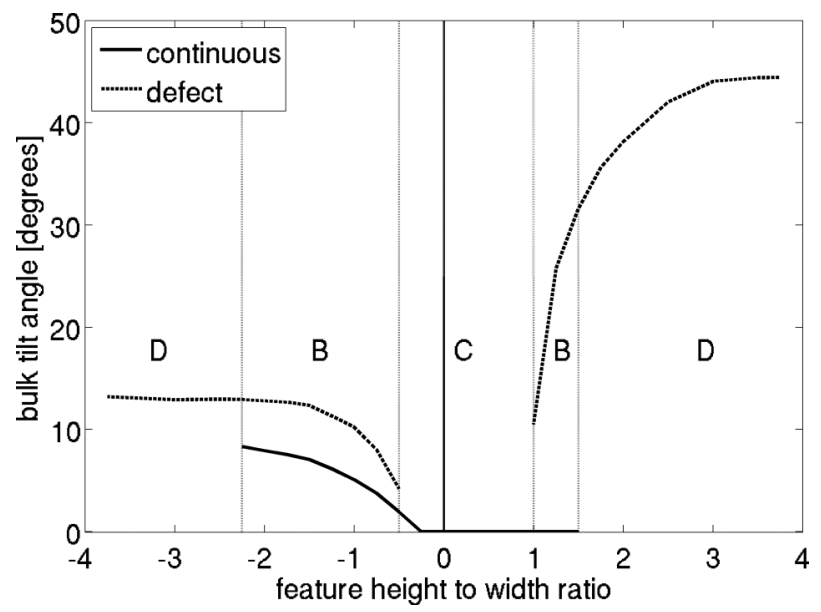

FIG. 5. Effective bulk tilt angle as a function of the feature aspect ratio $(d / w)$ for $K_{11}=K_{33}$.

low depths, very soon it becomes asymmetric as the depth is increased, before the discontinuous configuration appears. Both the continuous and discontinuous states are possible for a certain range of values of depths until only the discontinuous (defect) structure is stable. The letters $\mathrm{C}, \mathrm{D}$, and $\mathrm{B}$, in Figs. 4, 5, and 6 indicate the regions where the continuous state, the defect state, or both are stable. The behavior described not only depends on the geometry of the features but also on the material parameters of the liquid crystal. For example, if these are approximated using $K_{11}=K_{33}$, the situation changes as shown in Fig. 5. In this case we find that for arrays of posts, as the height is increased, the continuous symmetric state does not develop into a continuous asymmetric state as before but gives rise to the defect state directly.

Figure 6 shows the difference between the elastic energies of the continuous and defect states in both wells and posts in the bistability region. It can be seen that in both types of structures, if the depth (or height) is small compared to the width the continuous state is more stable but the situation changes when $d / w$ increases.

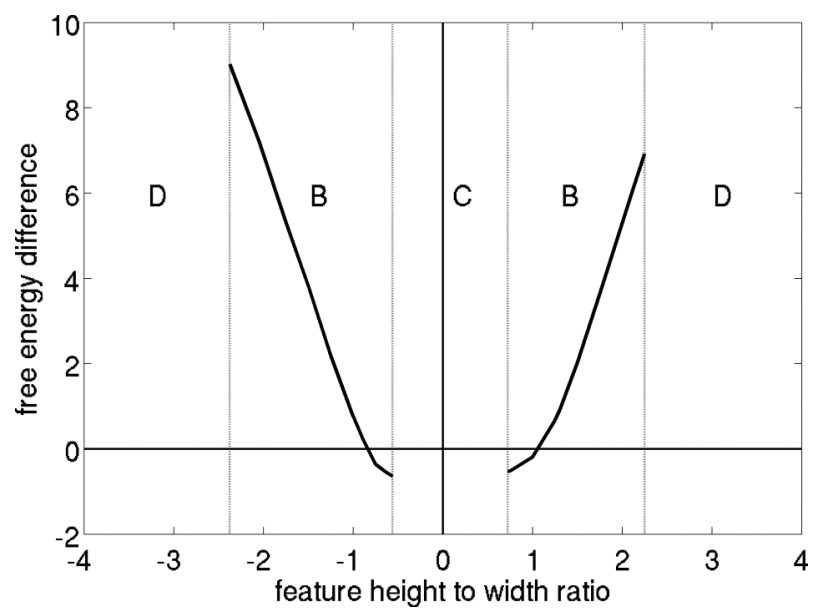

FIG. 6. Difference between the free energies of the continuous and defect states as a function of the aspect ratio $d / w$. 


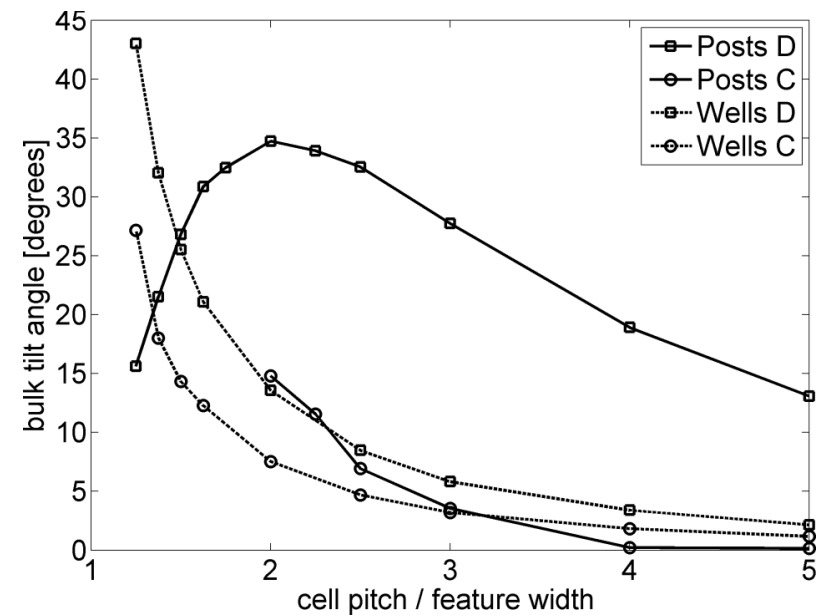

FIG. 7. Variation of bulk tilt angle as a function of feature separation using $5 \mathrm{CB}$ material parameters for the continuous $(\mathrm{C})$ and defect (D) states.

\section{A. Effect of feature separation}

The effect of the separation between posts or wells is investigated next. The features height or depth is fixed at $d=700 \mathrm{~nm}$ and the width at $w=400 \mathrm{~nm}$, while the period $p$ of the structure (or distance between centres and width of the modeling window) is varied. The value of $d / w$ is chosen so, in this case, both the defect and the continuous states, reached when starting from the initial high-tilt and low-tilt configurations, respectively, are stable. Figure 7 shows the variation of the induced tilt in the bulk when the period of the structure is varied. For arrays of wells, in both modes, the tilt in the bulk increases when the feature separation decreases. In the case of posts, an intermediate feature separation yields the highest bulk tilt angles, with both small and large feature periods favoring lower bulk tilt angles.

\section{B. Estimation of anchoring strength}

We investigate the effect of feature height on the effective anchoring strength of the patterned surface by comparing the average LC director orientations as functions of the $z$ coordinate (across the cell gap) within our simulated 3D cells with that in a simple one-dimensional cell with weak anchoring conditions represented by a Rapini-Papoular expression. The total energy for a 1D cell of thickness $d$ sandwiched between two aligning surfaces can be written as follows:

$$
F_{\text {tot }}=F_{d}+F_{s 1}+F_{s 2},
$$

where $F_{d}$ is the elastic distortion energy in the bulk and $F_{s 1}$ and $F_{s 2}$ are the anchoring energies on the bottom and top surfaces, respectively.

When restricted to a twist-free case, the total elastic distortion energy in the bulk of the cell can be given by $F_{d}(\Delta \theta)$, where $\Delta \theta$ is the total change in the polar angle within the cell, given by $\Delta \theta=\theta_{T}-\Delta \theta_{1}-\Delta \theta_{2} ; \theta_{T}=\theta_{\text {top }}-\theta_{0}$ is the difference between the easy polar angular directions on the two anchoring surfaces; and $\Delta \theta_{1}$ and $\Delta \theta_{2}$ are the angular deviations from the easy directions $\theta_{0}$ and $\theta_{\text {top }}$ at the respective surfaces as shown in Fig. 8(b).

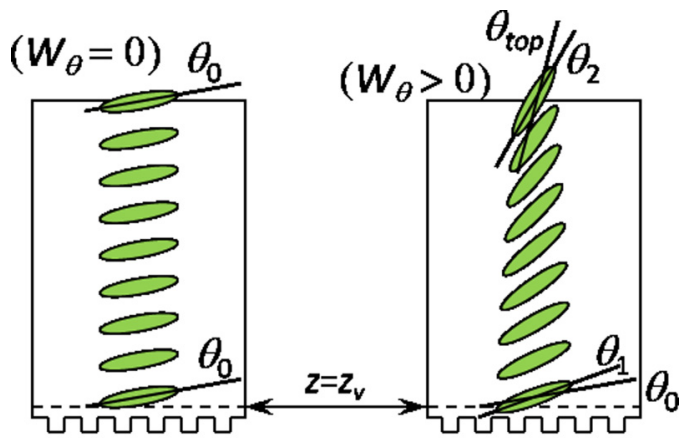

(a)

(b)

FIG. 8. (Color online) (a) The easy direction in the effective anchoring surface is found as the resultant alignment angle at the top when $W_{\theta}=0$. (b) Imposing an alignment $\theta_{\text {top }}$ with strength $W_{\theta}>0$ at the top results in alignments at angles $\theta_{1}=\theta_{0}+\Delta \theta_{1}$ at the bottom and $\theta_{2}$ at the top.

The steady-state LC configuration is that which minimizes $F_{\text {tot }}$ with respect to $\Delta \theta_{1}$ and $\Delta \theta_{2}$, and this results in the following:

$$
\frac{\partial F_{s 1}}{\partial \Delta \theta_{1}}=\frac{\partial F_{s 2}}{\partial \Delta \theta_{2}} .
$$

Modelling the anchoring energies on both surfaces by use of the Rapini-Papoular expression (8) leads to the following expression:

$$
W_{\text {eff }} \sin \left(2 \Delta \theta_{1}\right)=W_{\theta} \sin \left(2 \Delta \theta_{2}\right),
$$

where $W_{\text {eff }}$ is the the unknown effective anchoring strength at $z=z_{v}$ above the patterned bottom surface and $W_{\theta}$ is the known anchoring strength of the top surface.

A two-step procedure is then used in conjunction with Eq. (9) to evaluate the effective anchoring strengths, $W_{\text {eff }}$, for select $d / w$ ratios: First, values for the easy tilt angle and a "virtual surface" at a height $z_{v}$ are obtained as described earlier, using $W_{\theta}=0$ on the top surface. The virtual height of the effective anchoring surface is here taken as the height above the patterned surface where the tilt angle is within a $1^{\circ}$ tolerance from the easy tilt angle. Typically this occurs within a few hundred nanometers, depending on the feature height and whether defects are present. Then, a small zenithal distortion is introduced by choosing $W_{\theta}>0$ on the top surface and setting $\theta_{\text {top }}=\theta_{0}+\delta$, where $\delta$ is a small angle. No azimuthal distortion is introduced. The equilibrium configuration is again found numerically, and the angular deviations $\Delta \theta_{2}=\theta_{\text {top }}-\theta_{2}$ and $\Delta \theta_{1}=\theta_{1}-\theta_{0}$ are obtained, where $\theta_{1}$ is the average tilt angle on the effective anchoring surface at $z=z_{v}$.

The results are shown in Fig. 9, where the ratio $W_{\text {eff }} / W_{1}$ is plotted for different values of $d / w$ ( $w$ is chosen as $400 \mathrm{~nm}$ in each case), where $W_{1}$ is the strength of the planar degenerate anchoring on the patterned bottom surface. For the case of the flat surface, i.e., $d / w=0$, the effective anchoring strength is a few percentages higher than $W_{1}$. This is expected when expression (5) is used to represent the energy of a surface imposing planar degenerate anchoring, as described in Ref. [15]. As the feature height (depth) is increased, the effective anchoring strength rapidly decreases, reaching a steady strength approximately two orders of magnitude weaker 


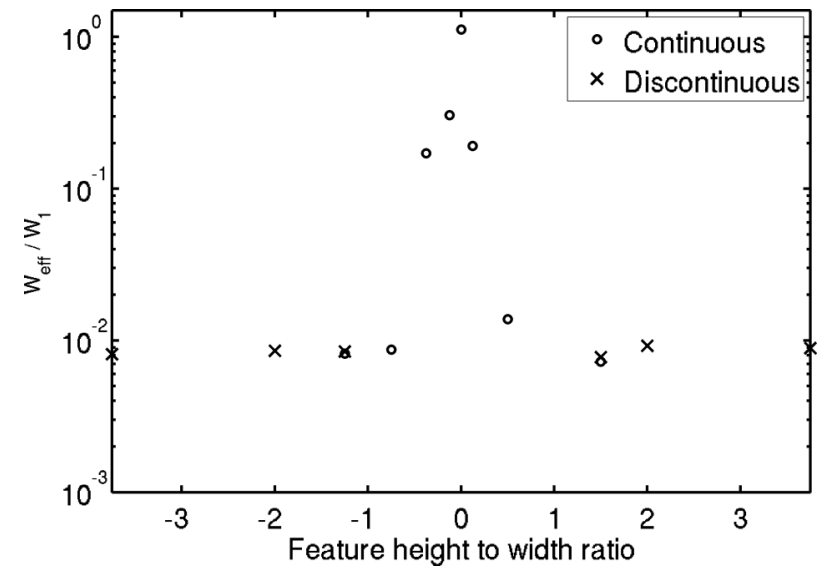

FIG. 9. Effective polar anchoring strength of patterned surface as a function of feature height.

than $W_{1}$ for features whose heights (depths) are equal or greater than their widths.

\section{ANALYSIS AND CONCLUSIONS}

A comprehensive three-dimensional modeling of the behavior of nematic liquid crystal material in contact with micropatterned surfaces has been presented, showing the effect of the surface pattern on the bulk alignment of the liquid crystal. The effect of changing the dimensions describing the profile of the surface features has been explored both for arrays of posts and of wells. It is found that for shallow wells and for short posts, a symmetric continuous state without defects is stable-this is consistent with the limit case of zero depth or height. For both types of patterns, as the depth of the wells or the height of the posts is increased, the orientation becomes asymmetric, leading eventually to the formation of defects. Both asymmetric states, with and without defects, induce tilt in the bulk. This is higher for the defect state and higher for posts than for wells of the same aspect ratio. In both cases there is a region of bistability where the continuous (symmetric or asymmetric) state can coexist with the high tilt defect state but for deep wells and tall posts only the defect state is stable. For both types of patterns, the induced tilt in the bulk also depends on the feature separation. The bulk tilt is found to vary close to the patterned surface but becomes uniform at a short distance from the surface pattern; this distance also depends on the feature separation.

The surface anchoring in these calculations was chosen as planar degenerate but sufficiently strong to prevent anchoring breaking. It is expected that if this anchoring is weak, the region of bistability in both structures will be reduced. The shapes studied were all of rounded square cross section. In these cases, the azimuthal alignment of the asymmetric states, continuous and defect, has a twofold degeneracy. This can be avoided with elongated shapes in the cross section, favoring one diagonal. The tilt degeneracy can be removed by changing the slope of the feature at the edges opposing the longer diagonal, which forces the asymmetric states to adopt a unique orientation.
[1] F. J. Kahn, Appl. Phys. Lett. 22, 386 (1973).

[2] B. Jérôme, Rep. Prog. Phys. 54, 391 (1991).

[3] A. V. Zakharov and R. Y. Dong, Phys. Rev. E 64, 042701 (2001).

[4] S. Ishihara, IEEE/OSA J. Display Technol. 1, 30 (2005).

[5] B. Zhang, F. K. Lee, O. K. C. Tsui, and P. Sheng, Phys. Rev. Lett. 91, 215501 (2003).

[6] S. C. Kitson, E. G. Edwards, and A. D. Geisow, Appl. Phys. Lett. 92, 073503 (2008).

[7] Y. W. Yi, V. Khire, C. N. Bowman, J. E. Maclennan, and N. A. Clark, J. Appl. Phys. 103, 093518 (2008).

[8] V. K. Gupta and N. L. Abbot, Science 276, 1533 (1997).

[9] S. Park, C. Padeste, H. Schift, J. Gobrecth, and T. Scharf, Adv. Mater. 17, 1398 (2005).

[10] T. Scharf, S. Park, C. Padeste, H. Schift, N. Basturk, and J. Grupp, Mol. Cryst. Liq. Cryst. 438, 55 (2005).

[11] J. P. Bramble, S. D. Evans, J. R. Henderson, C. Anquetil, D. J. Cleaver, and N. J. Smith, Liq. Cryst. 34, 1059 (2007).

[12] A. Rapini and M. Papoular, J. Phys. Colloques 30, C4 (1969).

[13] W. Zhao, C.-X. Wu, and M. Iwamoto, Phys. Rev. E 62, R1481 (2000).

[14] W. Zhao, C.-X. Wu, and M. Iwamoto, Phys. Rev. E 65, 031709 (2002).

[15] E. Willman, F. A. Fernandez, R. James, and S. E. Day, IEEE Trans. Electron. Dev. 54, 2630 (2007).
[16] S. C. Kitson and A. D. Geisow, Appl. Phys. Lett. 80, 3635 (2002).

[17] E. L. Wood, G. P. Bryan-Brown, P. Brett, A. Graham, J. C. Jones, and J. R. Hughes, SID Symposium Digest of Technical Papers 31, 124 (2000).

[18] Y. Yi, G. Lombardo, N. Ashby, R. Barberi, J. E. Maclennan, and N. A. Clark, Phys. Rev. E 79, 041701 (2009).

[19] G. P. Bryan-Brown, C. V. Brown, and J. C. Jones, U. S. Patent No. 6249332, 1995.

[20] C. Uche, S. J. Elston, and L. A. Perry-Jones, J. Phys. D: Appl. Phys. 38, 2283 (2005).

[21] W. Schenck, D.-H. Ko, and E. Samulski, J. Appl. Phys. 109, 064301 (2011)

[22] S. Kitson, U. S. Patent No. 6903790 B2, 2005.

[23] R. B. Meyer, Phys. Rev. Lett. 22, 918 (1969).

[24] E. Willman, F. A. Fernandez, R. James, and S. E. Day, IEEE J. Display Technol. 4, 276 (2008).

[25] L. Harnau, S. Kondrat, and A. Poniewierski, Phys. Rev. E 76, 051701 (2007)

[26] F. C. Frank, Discuss. Faraday Soc. 25, 19 (1958).

[27] C. W. Oseen, Trans. Faraday Soc. 29, 883 (1933).

[28] D. W. Berreman, Phys. Rev. Lett. 28, 1683 (1972).

[29] J. I. Fukuda, M. Yoneya, and H. Yokoyama, Phys. Rev. Lett. 98, 187803 (2007).

[30] J. I. Fukuda, M. Yoneya, and H. Yokoyama, Phys. Rev. E 79, 011705 (2009). 
[31] G. Barbero, A. S. Gliozzi, M. Scalerandi, and L. R. Evangelista, Phys. Rev. E 77, 051703 (2008).

[32] P. G. de Gennes and J. Prost, The Physics of Liquid Crystals (Oxford University Press, Oxford, 1993).

[33] R. James, E. Willman, F. A. Fernandez, and S. E. Day, IEEE Trans. Electron. Dev. 53, 1575 (2006).

[34] T. J. Spencer and C. M. Care, Phys. Rev. E 74, 061708 (2006).
[35] C. Anquetil-Deck, D. J. Cleaver, J. P. Bramble, and T. J. Atherton, Phys. Rev. E 88, 012501 (2013).

[36] S. Dickmann, J. Eschler, O. Cossalter, and D. A. Mlynski, SID Symposium Digest of Technical Papers 24, 638 (1993).

[37] H. Mori, J. E. C. Gartland, J. R. Kelly, and P. Boss, Jpn. J. Appl. Phys. 38, 135 (1999).

[38] H. J. Coles, Mol. Cryst. Liq. Cryst. 49, 67 (1978)..

[39] P. P. Karat and N. V. Madhusudana, Mol. Cryst. Liq. Cryst. 40, 171 (1977). 